\title{
A NEW SURVEY FOR H $\alpha$-EMISSION-LINE STARS AND RELATED OBJECTS IN THE SMC
}

\author{
M. MEYSSONNIER, M. AZZOPARDI \\ Observatoire de Marseille \\ 2, Place Le Verrier \\ 13248 Marseille Cedex 4 \\ France
}

ABSTRACT. New identifications of the H $\alpha$-emission-line objects in the Small Magellanic Cloud (SMC) have been made with the Curtis Schmidt telescope at Cerro Tololo Inter-American Observatory (CTIO).

Observations were carried in 1982 August 14-16, using the $10^{\circ}$ objective-prism giving a dispersion of $420 \AA / \mathrm{mm}$ at $\mathrm{H} \alpha$ and a $110 \AA$ bandwidth interference filter (5 inch square) with a peak wavelength at $6565 \AA$. Accordingly, the useful field was reduced to a $3.4 \mathrm{deg}$ square. Thanks to the $\mathrm{H} \alpha+[\mathrm{NII}]$ interference filter, exposures from $30 \mathrm{~min}$ to $4 \mathrm{hrs}$ on hypersensitized IIIa-F plates allowed us to survey (limiting $\mathrm{m}_{\mathrm{pg}} \sim 18$ ), even in the most crowded regions, the SMC emission-line objects up to 3 mag fainter than those previously found by Henize (1956) and Lindsay (1961).

$\mathrm{H} \alpha$-emission-line objects were searched for by carefully surveying our set of plates with a binocular microscope, as well as by a systematic examination of displayed images on screen, obtained from the full PDS microdensitometer scan of the 4-hr exposure objective-prism plate. The latter technique was especially useful for confirming the faintest emission-line objects, estimating the relative intensity and the shape of emission lines and for measuring accurate positions ( 0.5 arcsec rms) for the objects of interest.

Now completed, this new SMC survey resulted in the identification of $1898 \mathrm{H \alpha}$-emission-line objects, almost quadrupling the number of those found, in the same area, by Lindsay's systematic survey (499 objects, but only 462 confirmed by us). A catalogue, providing coordinates, description of the spectral features, cross-identifications with other lists, as well as $2 \times 2$ arcmin finding charts for our complete sample emission objects will be published soon. Most of the objects (1742) we have detected are more likely $\mathrm{H} \alpha$-emission-line stars. Among the remaining objects, 45 are compact HII regions and 65, planetary nebulae (PN); 18 are new candidates that escaped previous PN surveys. Subsequent medium resolution slit spectroscopy allowed us to confirm the PN or VLE nature of 15 of the new objects that we discovered (see Azzopardi \& Meyssonnier 1988).

Fig. 1 presents surface distributions of both our $\mathrm{H} \alpha$-emission-line star and our PN samples. The emission-line star distribution shows strong concentration zones in the SMC bar, more extended in its NE part than in the SW, as well as in the so-called "wing" (region of the young clusters NGC 456, 460 and 465). Although less numerous, the early- and late-supergiant stars display the same overall pattern (see Fig. 3 of Azzopardi \& Rebeirot, 1990). However, there are emission-line stars in the Eastern and Northern peripheral regions of the bar, which is not the case 


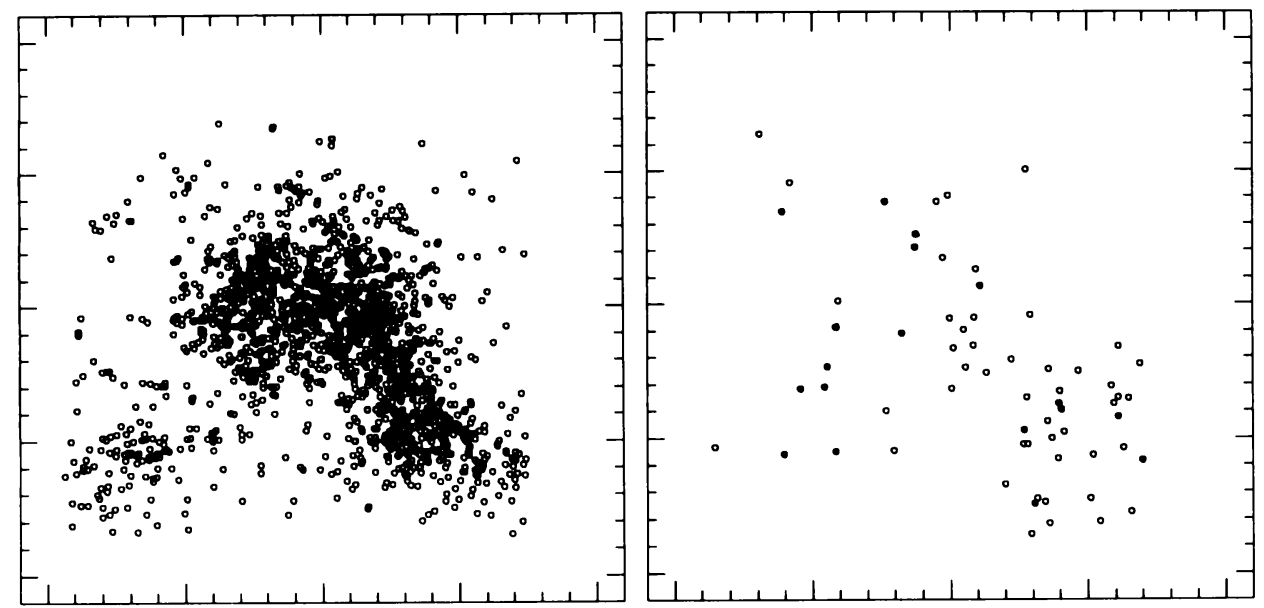

Figure 1. Surface distributions of $1742 \mathrm{H} \alpha$-emission line stars (left panel) and $65 \mathrm{PN}$ (right panel); filled circles indicate the new PN or PN candidates we have discovered.

for supergiant stars. At this stage, it is difficult to know if it is a real structure or an observing bias. In spite of the restricted sample, the PN surface distribution shows this trend in part since some of those objects must be of type I, thus relatively young (a few $10^{8}$ years).

More recently, in the framework of the ESO key program "Coordinated Investigation of Selected Regions in the Magellanic Clouds", we have undertaken a very deep survey of $\mathrm{H} \alpha$ and/or [NII]-emission-line objects, among other objects of interest, in two 30x30 arcmin SMC fields. For this purpose, CCD observations have been performed, in the slitless spectroscopy mode, with the ESO Faint Object Spectrograph and Camera (EFOSC) at the Cassegrain focus of the $3.6 \mathrm{~m}$ telescope, using a grism with $270 \AA / \mathrm{mm}$ dispersion and a $\mathrm{H} \alpha+[\mathrm{NII}]$ interference filter $\left(\lambda_{0}=6569 \AA\right.$; $\Delta \lambda=110 \AA$ ). Integrations of $15 \mathrm{~min}$ allowed us to reach objects at the limiting magnitude $R \sim 20$. Thanks to special software routines, data reduction is under way; preliminary results show that the technique is very powerful. Indeed, in the very young cluster NGC 330 we find $61 \mathrm{H \alpha}-$ emission-line stars, while only 10 were identified by Feast (1972) in the same area. Furthermore, we have discovered in the surrounding field about twice as many new $\mathrm{H} \alpha$-emission-line objects as in our previous Schmidt survey. The result of this is that a rough estimate of the total number of $\mathrm{H} \alpha$-emission-line objects of the SMC may be about 4000 !

\section{References}

Azzopardi, M., Meyssonnier, N. (1988), Astron. Soc. Pacific Conf. Ser., Vol. 1, V.M. Blanco, M.M. Phillips (eds.) p.386.

Azzopardi, M., Rebeirot, E. (1990), these proceedings.

Feast, M.W. (1972), Mon. Not. R. Astron. Soc. 159, 113.

Henize, K.G. (1956), Astrophys. J. Suppl. Ser. 2, 315.

Lindsay, E.M. (1961), Astron. J. 66, 169. 